\title{
The FIRST-APM QSO Survey (FAQS) in the SBS Region. Current Status
}

\author{
V. Chavushyan, R. Mújica, J.R. Valdés, L. Carrasco \\ INAOE, A.P. 51 y 216. C.P. 72000. Puebla, Pue., México. \\ J. Stepanian \\ IA-UNAM, A.P. 70-264, México D.F. 04510, México. \\ O. Verkhodanov \\ SAO RAS. Karachai-Cherkesia, 357147. Russia.
}

\begin{abstract}
As the first step of the Multiwavelength AGN Survey (MWAS), we have started the FIRST-APM QSO Survey (FAQS). The main goal of FAQS is to compile the most complete sample of bright QSOs, located in the area of the sky covered by the Second Byurakan Survey (SBS). Here we report the current status of an ongoing study based on the crossidentification of the FIRST radio catalog and the APM optical catalog. The overlapping sky area between FIRST and SBS is about $700 \mathrm{deg}^{2}$. The compiled list of sources for this overlapping region contains $\sim 400$ quasar candidates brighter than $B=18 \mathrm{~m} .5$. About 90 objects were already spectroscopically classified. During 1999-2000, we observed spectroscopically more than 150 FAQS objects with the $2.1 \mathrm{~m}$ telescope of the Guillermo Haro Astropysical Observatory (GHAO). We have found 51 new QSOs (4 BAL QSOs), 13 Seyfert Galaxies (5 NLSy1's), 23 emission line galaxies, 3 BL Lac objects and 57 stars.
\end{abstract}

\section{Introduction}

Every survey technique has redshift and luminosity-dependent selection biases (Wampler \& Ponz 1985). Taking into account the selection effects inherent to every technique adopted to search for QSOs, it is obvious that only a combination of different search techniques in different spectral ranges will yield a complete sample of quasars with an adequate representation of all the properties inherent to them (Hartwick \& Shade 1990).In order to circumvent the problem of bias, we have started a multi-wavelength search for QSOs in the well investigated Second Byurakan Survey sky area (Stepanian et al. 1999, 2001).

As the first step of the Multiwavelength AGN Survey (see Stepanian \& Chavushyan, contribution to this colloquium), we cross-identified the FIRST (Becker et al. 1995) radio and APM optical catalogs. The FIRST radio survey provides a new resource for constructing a large quasar sample (White et al. 2000 ). However, the drawback of radio selection is that most of the radio-quiet 
quasars will not be included in the sample. Therefore, the sample will not be a good representation of the quasar population. An example is that only $25 \%$ of SBS QSOs were detected in the FIRST survey.

\section{Sample, Observations, and Preliminary Results}

We named this sample FAQS - FIRST-APM Quasar Survey (Chavushyan et al. 2001). The overlapping area between the FIRST and SBS surveys is quite significant, covering about $700 \mathrm{sq}$. degrees, within the limits defined by $07^{h} 40^{m}<$ $\alpha<17^{h} 15^{m}$, and $+48^{\circ} 52^{\prime}<\delta<+57^{\circ} 36^{\prime}$. The cross-identification between the FIRST and APM catalogues was done adopting the following selection criteria: a) position coincidence between FIRST and APM objects within $3^{\prime \prime}$ radius; b) Stellar-like classification on APM; c) B-magnitude between 14.5 and 18.5 on APM. The resulting list of sources, in the overlapping region, contains 412 objects, 90 of which were previously known AGNs, mainly discovered by the SBS.

The spectroscopy was carried out with the $2.1 \mathrm{~m}$ telescope of the Guillermo Haro Astropysical Observatory (INAOE-Mexico) and the LFOSC focal reducer (Zickgraf et al. 1997). A set-up covering the spectral range 4200-9000 A, with a dispersion of $8.2 \AA$ /pixel was adopted. The effective instrumental spectral resolution was about $16 \AA$. During 1999-2001, we have carried out spectroscopic observations for $\sim 150$ FAQS objects. The BV photometry for the objects classified as AGN $(B<17.5)$ was carried out with the $1.5 \mathrm{~m}$ telescope of San Pedro Martir Observatory (UNAM-Mexico), and reduction is in process.

In the subsample studied until now, we have found 51 new QSOs, 13 Seyfert Galaxies (5 NLSy1's), 23 emission line galaxies (ELG), 3 BL Lac objects and 57 high galactic latitude stars. Amongst the 51 QSOs, we have found 4 broad absorption line (BAL) QSOs.

We expect to complete spectroscopy and photometry of the total sample by the end of 2001.

Acknowledgments. This work was supported by CONACyT research grants 28499-E, J32178-E and, G28586-E.

\section{References}

Becker, R.H., White, R.L., \& Helfand, D.J., 1995, ApJ, 450, 559

Chavushyan, V.H. Mujica, R., Carrasco, L., Valdes, J.R., Verkhodanov, O., \& Stepanian, J., 2001, ASP Conf. Ser., 232, 102.

Hartwick, F.D.A., \& Shade, D., 1990, ARAA, 28, 437

Stepanian, J.A., Chavushyan, V.H., Carrasco, L., Tovmassian, H.M, \& Erastova, L.K., 1999, PASP, 111, 1099

Stepanian, J.A., Green, R.F., Foltz, C.B., Chaffee, F., Chavushyan, V.H., Lipovetsky, V.A., \& Erastova, L.K., 2001, AJ (in press).

Wampler, E.J. \& Ponz, D., 1985, ApJ, 298, 448

White, R.L., Becker, R.H., Gregg, M.D, et al., 2000, ApJS, 126, 133

Zickgraf, F.J., Thiering, I., Krautter, J. et al., 1997, A\&AS, 123, 103 